\title{
Cast Partial Denture Retained Using Precision Attachment - A Case Report
}

\author{
GulshanKumarTomar ${ }^{1}$,Mirna Garhnayak ${ }^{2}$, SitansuSekhar Das $^{3}$, Shelly Roy ${ }^{4}$ \\ ${ }^{I}$ Post Graduate Trainee, Department Of Prosthodontics, Ids, Bhubaneswar \\ ${ }^{2}$ P.G.Guide,Professor, Department Of Prosthodontics, Ids, Bhubaneswar \\ ${ }^{3}$ Hod, Professor, Department Of Prosthodontics, Ids, Bhubaneswar \\ ${ }^{4}$ Bds, Ids, Bhubaneswar
}

\begin{abstract}
The most challenging job for a prosthodontist is to rehabilitate the partial edentulous patient especially distal extension cases where fabrication of fixed prosthesis is quite impossible without using implants. Several conventional and contemporary treatment options were available for treating long span edentulous situations. For such cases, attachment retained cast partial dentures would be the best solution as they give economically, functionally as well as esthetically good results. This article provides a simplified approach for rehabilitating the partially edentulous situations using precision attachment via case report.
\end{abstract}

Keywords: Precision attachment, Cast partial denture, Removable partial denture

\section{Introduction}

The selection of prosthesis for rehabilitating partially edentulous mouth is basically dependent on extent of edentulous region, position of edentulous space, abutment teeth ability to resist occlusalload, mucosa as well as underlying residual bone. ${ }^{1}$ Rehabilitation of distal extension situations can be done by several conventional methods out of which one treatment modality is implant retained prosthesis but due to insufficient bone as well as for economic reasons patients were unable to accept that option. Alone fixed prosthesis for distal extension situations is not possible due to loss of natural teeth. Another treatment modality for such clinical situations is combination of fixed and removable partial dentures using precision attachment. This type of prosthesis not only gives esthetics in addition also gives functional advantage of fixed denture that leads to decreased compression of edentulous ridge and enhanced phonetics \& mastication. ${ }^{2}$

In this case, extra coronal attachment is chosen as all of its mechanism is outside of abutment periphery, less tooth preparation needed thus less chances of tooth devitilization.In this type of attachment, crown is made with the projecting attachment and a corresponding fitting or housing is incorporated in the removable prosthesis as a result, there is a certain amount of movement between the two sections of the prosthesis that acts as a non-rigid stress breaker and helps in distributing the occlusal load without breaking the prosthesis. $^{3}$

\section{Classification of Attachment}

Attachment is a connector consisting of two or more parts. One part is connected to a root, tooth or implant and the other part to prosthesis. Precision attachments can be classified in to four main groups ${ }^{4}$.

1. Intracoronal attachments are mainly used in connecting units of fixed partial prostheses, retaining restorations with distal extension or bounded removable prostheses.

2. Extracoronal attachments - this type of attachment provides stability and retention for removable distal extension prostheses.

3. Stud attachments - usually in the form of ball and socket, this attachment serves primarily for overdenture stabilization and retention of the prosthesis. Swiss logic, ZAAG, Zest anchor is example of stud attachments. One of the advantages of stud is that they promote better oral hygiene and crown-root ratio is improved with low profile stud attachments.

4. Bar attachments - originally used for splinting groups of teeth, currently used for overdenture retention and stabilization.

\section{Case Report}

A 51 year old female(Fig.1) patient reported to the department with a chief complaint of missing teeth in the upper jaw (back teeth on both sides) and lower jaw (back teeth on right side)(Fig.2) and wishes to replace the same. On clinical examination 14,13,12,11,21,22,23 were present (Fig. 3) in upper jaw \& 36, 46,47 were missing in lower jaw. Remaining teeth were periodontally sound and can be utilized as an abutment for precision attachment .Treatment was planned as precision attachment retained cast partial denture for both 
maxillary and mandibular arch.An informed and written consent was obtained from the patient prior to begin the treatment.

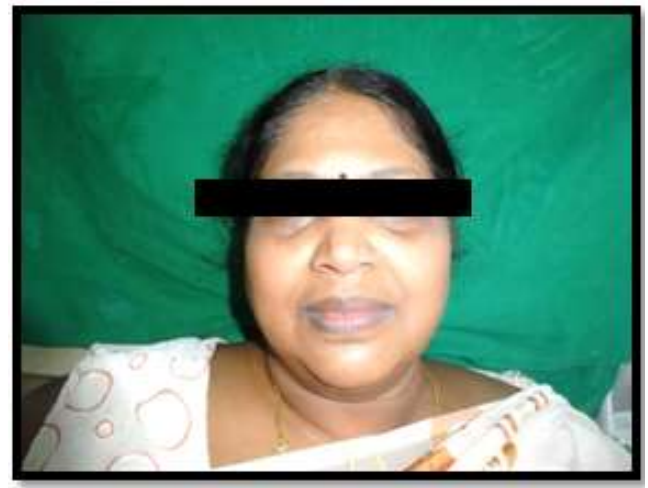

Fig.1 Preoperative Photograph

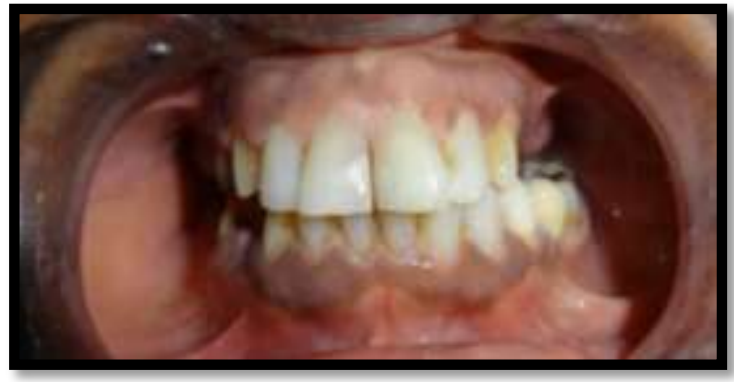

Fig.2 Intra-oral Photograph

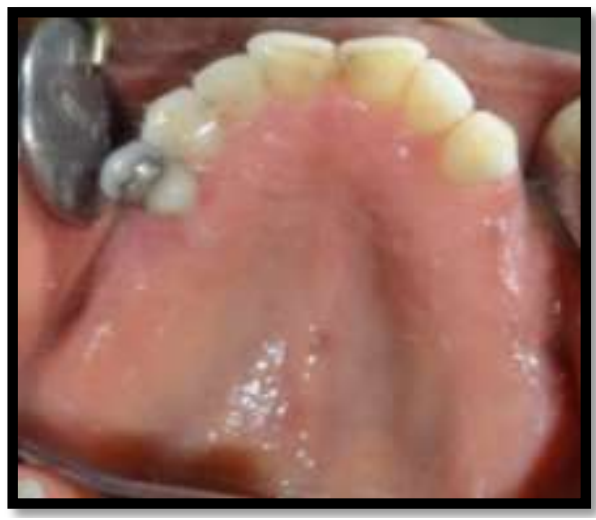

Fig.3 Maxillary Arch

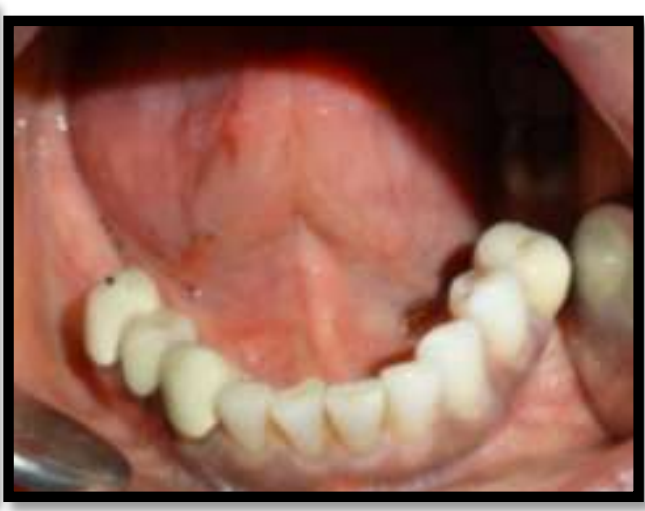

Fig.4 Mandibular Arch

\section{Technique ${ }^{5}$}

1. Diagnostic impressions were made and mounted on semi adjustable articulator using a face bow. Following which diagnostic wax-up was done on the mounted casts. A putty matrix (Express STD Putty; 3M ESPE, St. Paul, Minn.) was made over the completed diagnostic wax-up for evaluation of the existing space for the extracoronal resilient attachment. The attachment system was selected on the basis of available space. (OT CAP, Rhein 83 Inc, USA) (Fig 5 \&Fig 6). 

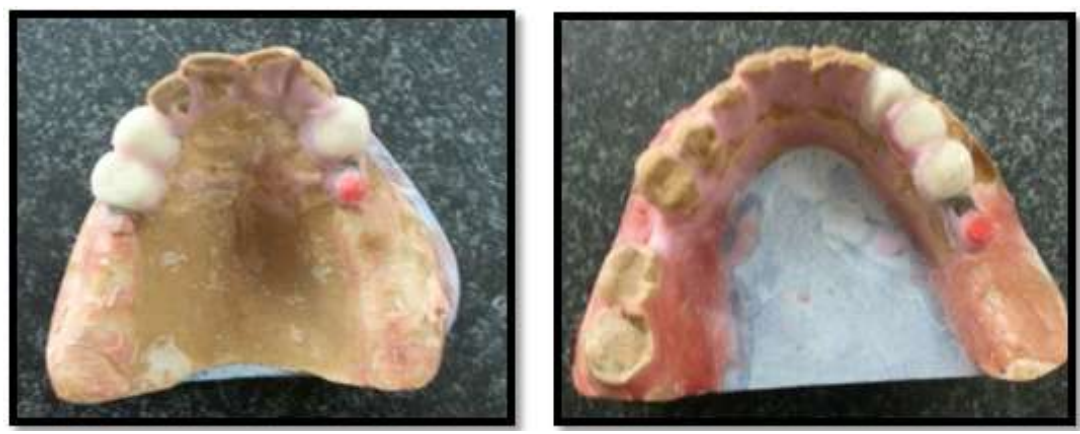

Fig.5- OT Cap in MaxillaFig.6-OT Cap in Mandible

2. Tooth preparations was done on $13,14,23,43,45$ to receive PFM crowns (Fig7 \& Fig 8). Impression was made and poured in die stone. Following which crowns have been waxed to full contour and milled in wax for maximum guiding plane surface. The Patrices was added to the axial surfaces of the abutment using a dental surveyor, lingual to the Centre of proximal contour. This ensures that the bulk of matrice does not interfere with esthetic of buccal cusp of replacing denture tooth. (Fig 9 \& Fig 10) .

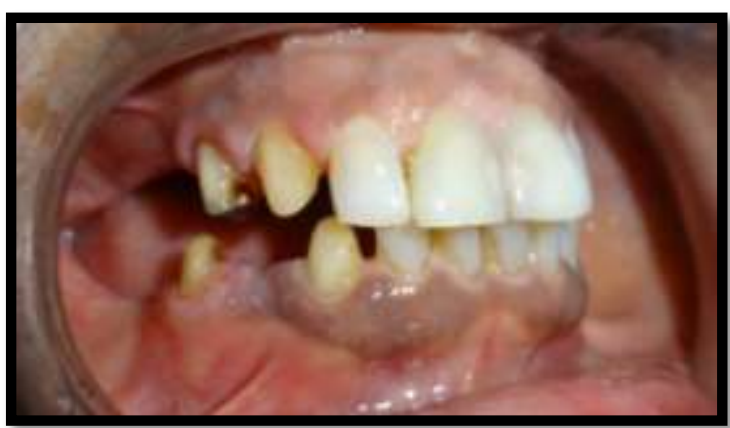

Fig. 7- Tooth Preparations on Right side

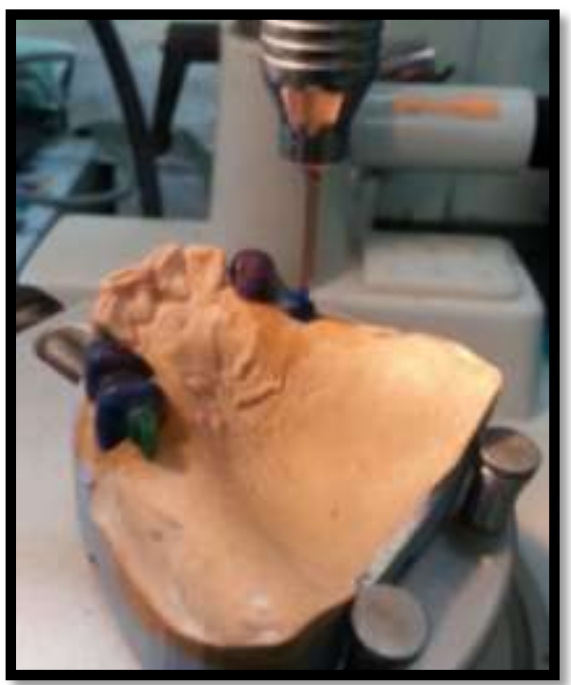

Fig.9 Milling done on maxillary cast

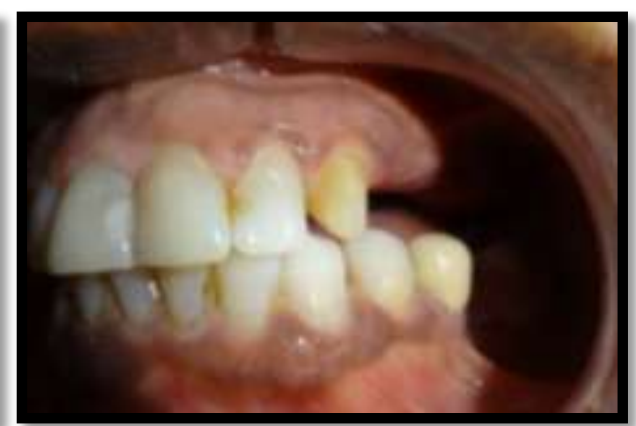

Fig.8-Tooth Preparations on Left side

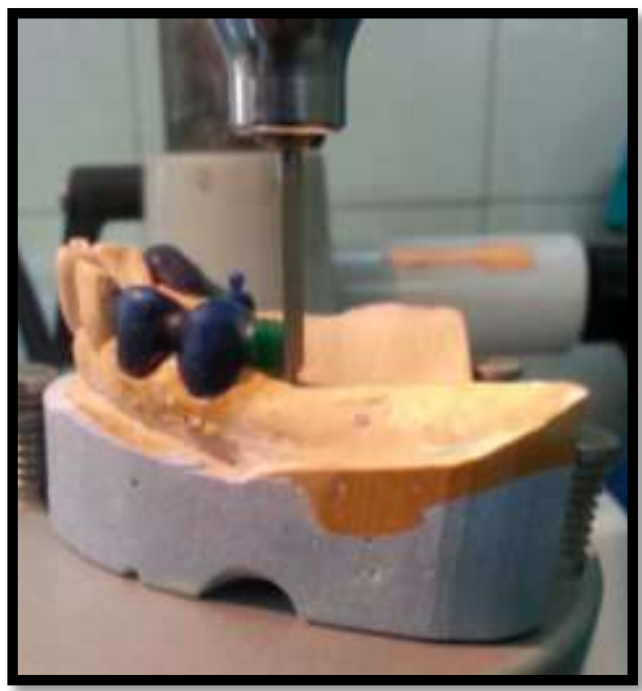

Fig.10-Milling done on mandibular cast

3. Following which casting, finishing, and veneering of the fixed component was done. (Fig: $11 \&$ Fig 12). 


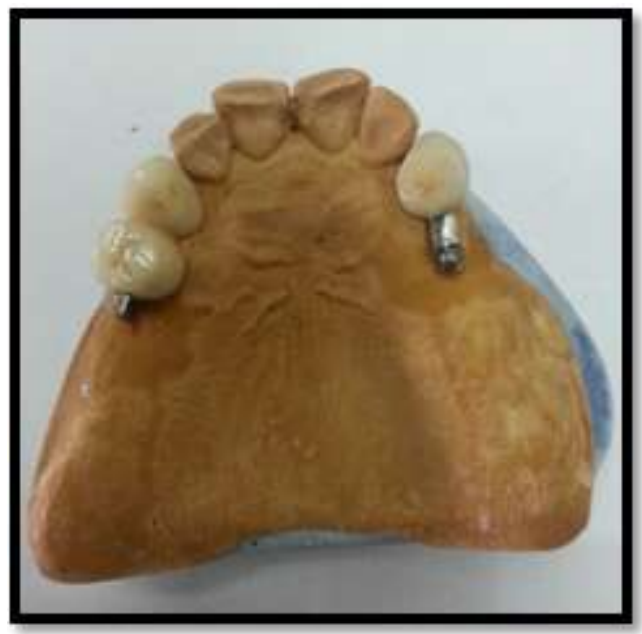

Fig.11-Crowns with attachment on maxillary cast

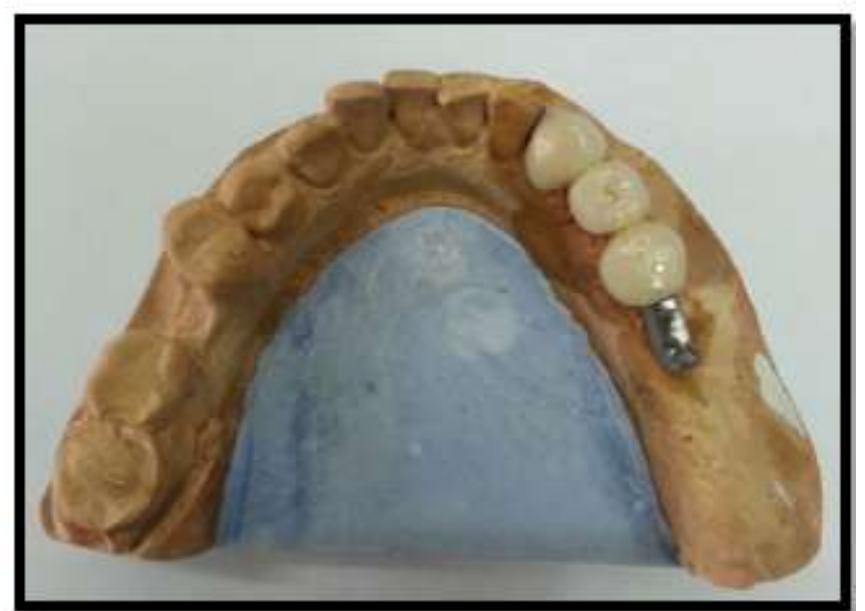

Fig.12-Crowns with attachment on mandibular cast

4. The fixed component including veneered metal-ceramic crowns \& the patrices were tried in the patient mouth ( Fig: $13 \&$ Fig 14) and a pick-up impression was made (Imprint II; 3M ESPE).
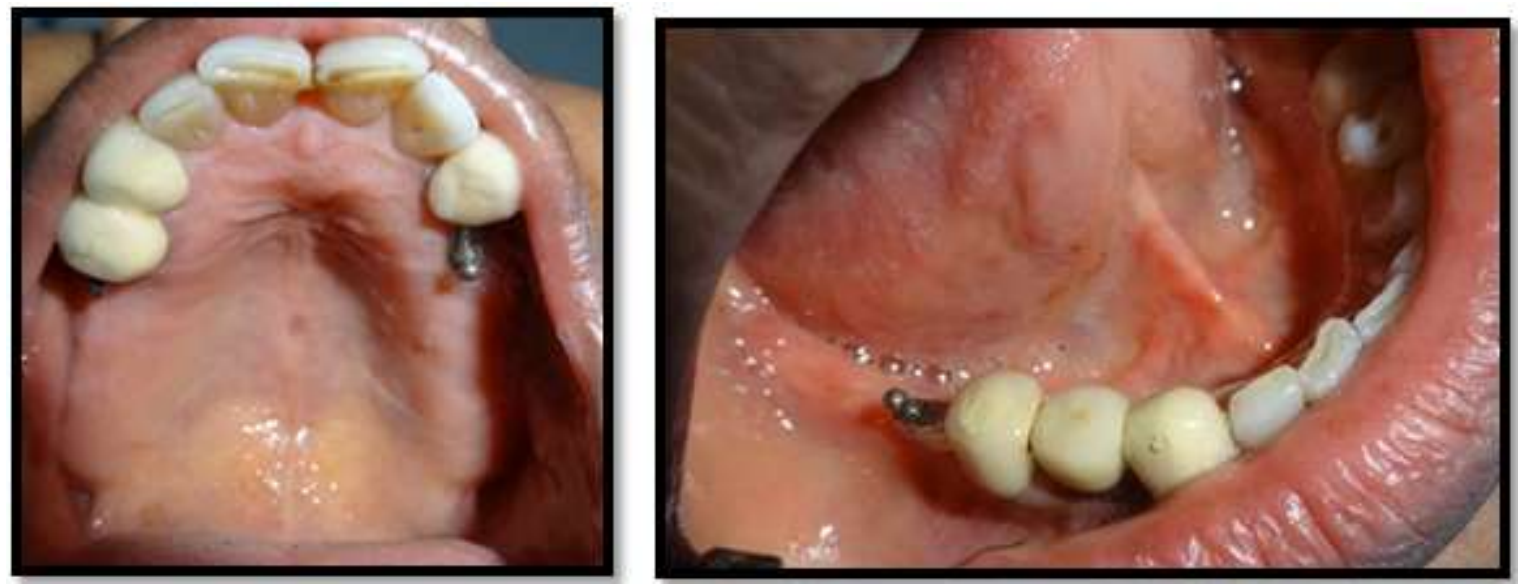

Fig.13- Bisque Trial in MaxillaFig. 14- Bisque Trial in Mandible

5. The matrices of the attachments was placed in the receptacles ( Patrice of the attachments) which were in the crowns on the refractory cast.(Fig $15 \&$ Fig 16).
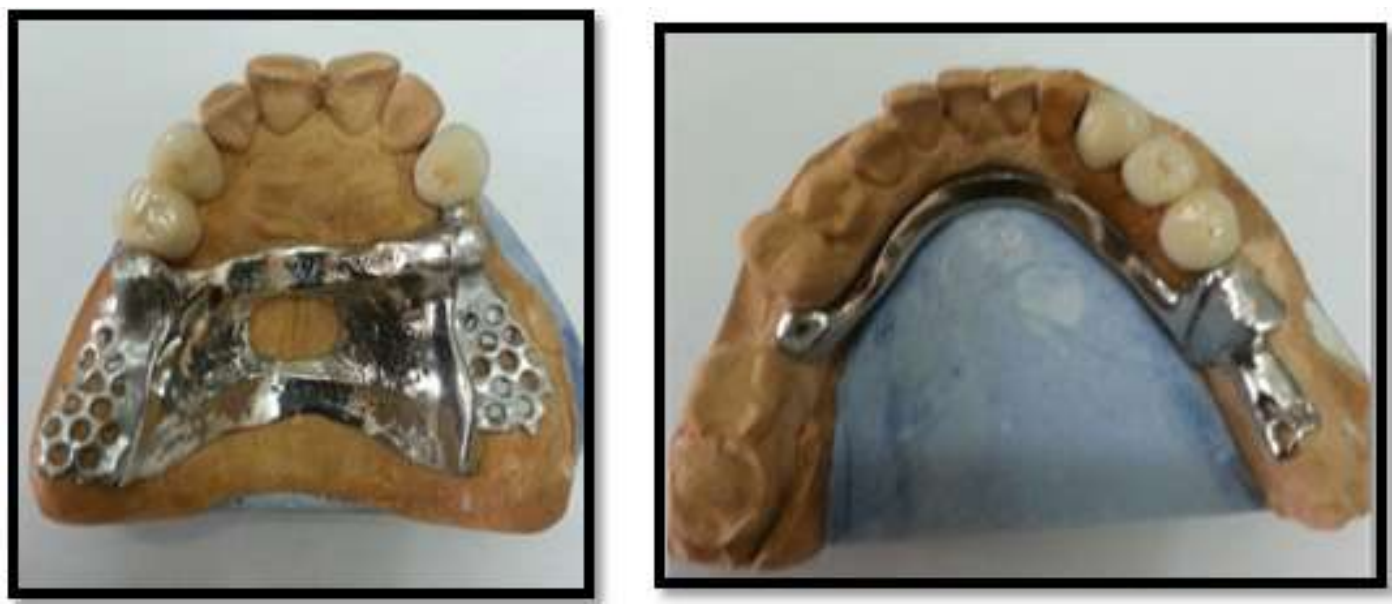

Fig.15- Metal Framework in MaxillaFig.16- Metal Framework in Mandible 
6. The wax up of framework of the removable partial denture was done, invested and casted. The framework was evaluated in the patient mouth .Try-in was done and acrylisation of removable partial denture was performed.
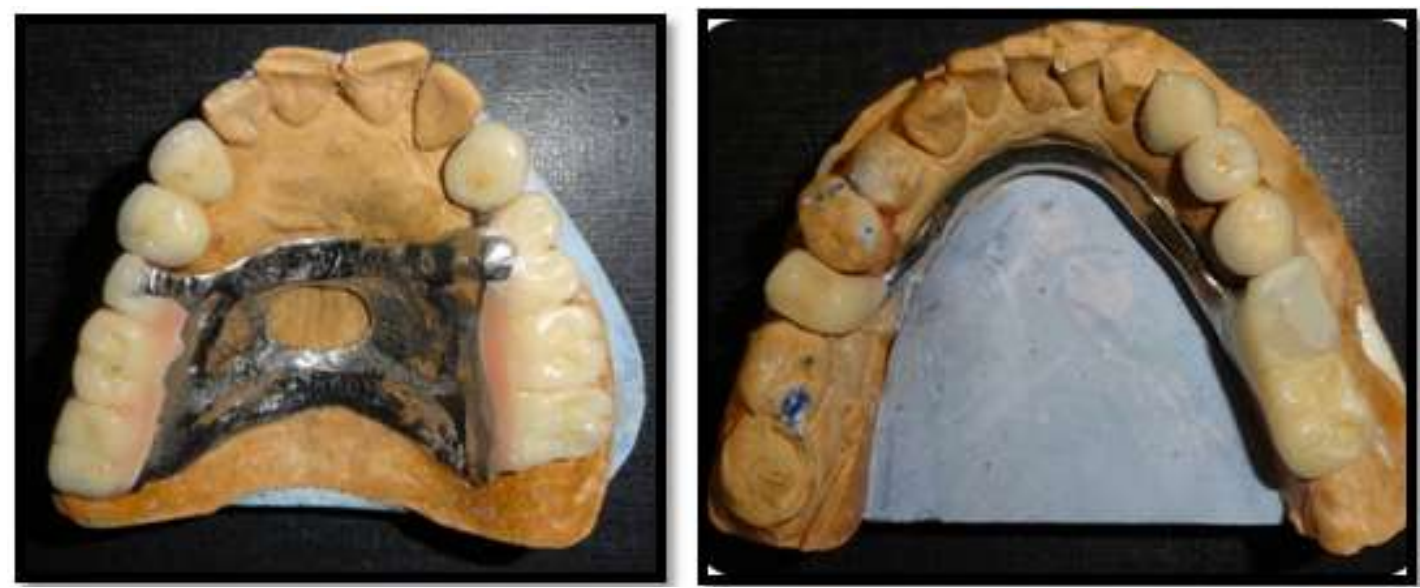

Fig.17-Acrylisationdone for maxillary frameworkFig.18-Acrylisation done for mandibular framework

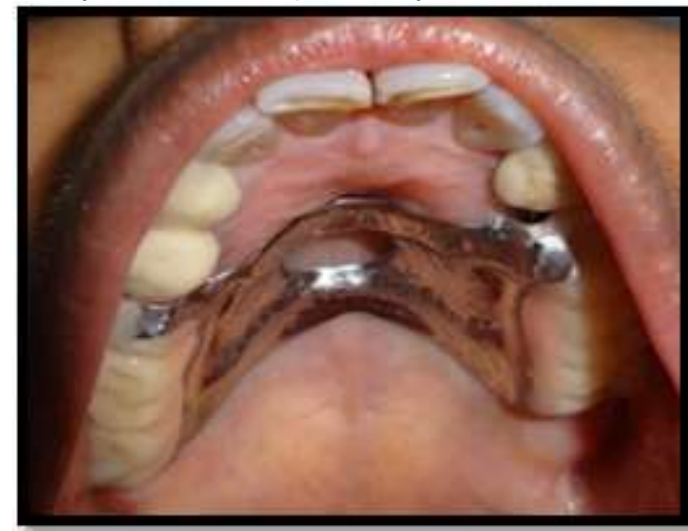

Fig.19-Intraoral view of maxilla

7. Outcome of Treatment

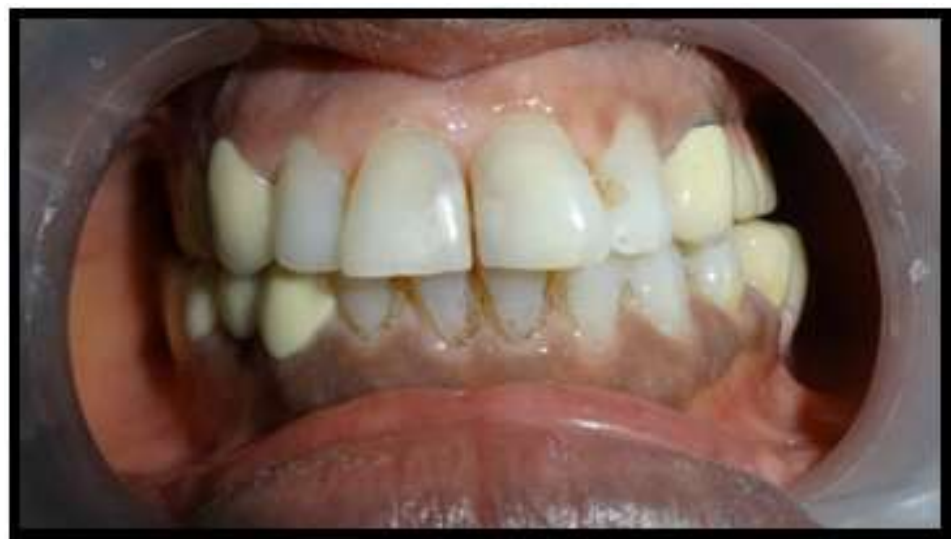

Fig.20-Frontal view Post operative

\section{Discussion}

Precision attachment provides the exceptional feature of improved retention, improved comfort \& less postoperative adjustments to the cast partial denture. Usually indicated for long spanedentulous arches, distal extension bases, and nonparallelabutments ${ }^{6}$.

The desire to balance between functional stability and cosmetic appeal in partial dentures gave rise to the development of Precision Attachments. Sometimes precision attachment is said to be a connecting link between fixed and removable prosthesis as it incorporates features of both type of prosthesis. The decision 
to use an intracoronal or extracoronalattachment should be based on the size and shape of the abutment teeth. Intracoronal attachments require more tooth preparation and tooth reduction than extracoronal attachments. If there is insufficient space intra coronal attachment is used, the abutment retainer will be overcontoured on the proximal surface, resulting in a restoration that can create periodontal problems. ${ }^{7}$

The customised attachments can overcome the disadvantages those which are associated with intracoronal attachments as (1) excessive tooth reduction; (2) compromised embrasures; and (3) poor esthetics. As well as they give the broad range for choosing alloys and easy handling. ${ }^{8}$

Most of the studies have shown that attachment retainedcast partial dentures gives better comfort, function, esthetics, less adjustments, protect abutment teeth, easy to clean and can Worn most of the time by the patient. Its use in fixed prostheses, overdentures and in implant therapy contributes to the success of prosthesis in terms of esthetics, comfort and function. ${ }^{9}$

\section{Conclusion}

When estheticsis of prime concern and economic condition does not permit the use of dental implants thenprecision attachment retained cast partial dentureswould be an excellent option for such cases. The stress control on abutment is an essential factor for the success of distal extension cast partial denture which is achieved through accurate impression technique, broad coverage and stable denture base, rigid design, physiologic shimming, splinting of abutments, proper selection of attachment. Precision attachments serve the function of retention, stress distribution and aesthetics successfully provided the case is planned based on sound biological and technical grounds and proper care is rendered by the dentist and the patient during the maintenance phase. The knowledge of material science, different attachments \&biomechanics of maxillomandibular function is very much essential in treating case using precision attachment. With proper case selection and treatment planning precision attachments are the viable option and can improve retention, esthetics and function.

\section{References}

[1]. JainD, Gupta A, ChhabraS,SangwanA. Prosthodontic Rehabilitation using Attachment Retained Overdenture- Case ReportsIOSRJDMS2015:14 (8) Ver. II):17-23.

[2]. Jain AR, Philip JM, Ariga P. Attachment-retained Unilateral Distal Extension (Kennedy's Class II Modification I) Cast Partial Denture. Int J ProsthodontRestor Dent 2012; 2(3):101-107.

[3]. Patel H, Patel K, ThummerS,PatelK.R.Use of precision attachment and cast partial denture for long-span partially edentulous mouth - A case reportIJADS 2014; 1(1): 22-25.

[4]. Preiskel HW. Precision attachment in prosthodontics.1\&2.London: Quintessence Publishing Co Ltd 1995:69-78

[5]. MakkarS,Chhabra A, KhareA. Attachment Retained Removable Partial Denture: A Case Report IJCDS: 2011:2(2).

[6]. WangooA,Kumar S, Phull S, Gulati M.Prosthetic Rehabilitation Using Extra Coronal Castable Precision Attachments IJDS :2014 Supplementary :6(4).

[7]. ReddyR,Thumati P.Prosthodontic Rehabilitation of Different Clinical SituationInternational Journal of Scientific and Research Publications 2014:4(1).

[8]. Shetty N, Shetty S, Shetty O, D’Souza. Precision Attachments for Aesthetics and Function: A Case ReportJournal of Clinical and Diagnostic Research. 2014: Vol-8(1): 268-270.

[9]. Shakeel SKRemovable prosthesis using extra coronal precision attachment: A case reportGMJ, ASM 2013; 2(S1):S126-S129. 